\title{
FDTD Study on Back Scattering of Conducting Sphere Coated with Double Negatibe Metamaterials
}

\author{
Maoyan Wang • Debiao Ge $\cdot$ Jun Xu $\cdot$ Jian Wu
}

Published online: 7 June 2007

(C) Springer Science + Business Media, LLC 2007

Erratum to: International Journal of Infrared and Millimeter Waves, Vol. 28, No. 2, February 2007

DOI: $10.1007 / \mathbf{s 1 0 7 6 2 - 0 0 6 - 9 1 9 1 - 7}$

The correct affiliation for Maoyan Wang is as follows:

School of Physical Electronics, University of Electronic Science and Technology of China, Chengdu Sichuan 610054, People's Republic of China.

The online version of the original article can be found at http://dx.doi.org/10.1007/s10762-006-9191-7

M. Wang $(\bowtie) \cdot$ J. Xu

School of Physical Electronics, University of Electronic Science and Technology of China, Chengdu Sichuan 610054, People's Republic of China

e-mail: wmybrimlhl@163.com

D. Ge

Physics Department, Xi'dian University,

Xi'an shanxi 710071, People's Republic of China

J. Wu

National Key Laboratory of Electromagnetic Environment,

Beijing 102206, People's Republic of China 